\section{Is Chlamydia pneumoniae infection a risk factor for age related macular degeneration?}

\author{
O Ishida, H Oku, T Ikeda, M Nishimura, K Kawagoe, \\ K Nakamura
}

The association between a pathogen and $A M D$ is worth further investigation because a new approach might treat this incurable vision threatening disease

A ge related macular degeneration (AMD) is a leading cause of decreased central vision in older people throughout the world. The pathogenesis of AMD is very complex and has still not been determined. In addition to some genetic and environmental factors, several kinds of risk factors have been proposed; sunlight exposure, ${ }^{1}$ smoking, ${ }^{2}$ and low levels of nutritional components such as antioxidants. ${ }^{3}$ Hypertension, ${ }^{4}$ hyperlipidaemia, ${ }^{4}$ and atherosclerosis, ${ }^{56}$ which may lead to cardiovascular diseases, are also considered to be risk factors.

Chronic inflammatory events have recently been identified as plausible causes of atherosclerosis. In particular, much interest has been focused on infections by Chlamydia pneumoniae, which was previously known as the TWAR strain. This strain is one of the chlamydial species that has been recognised as a causal mediator of respiratory infections such as bronchitis, pneumonia, and upper respiratory tract infections. $^{7}$

Chlamydia can multiply in various host cells including macrophages and endothelial cells. ${ }^{8}$ C pneumoniae is like a parasite and consumes energy that is needed by the host cells and, in the end, destroys them and then infects nearby cells. Thus, the pathogens tend to cause a chronic infection.

The first study showing a positive interaction between systemic vascular disease and infection with $C$ pneumoniae was made by Saikku et al in 1988. ${ }^{9}$ They reported increased immunoglobulin G (IgG) and IgA antibody titres against $C$ pneumoniae in male patients with myocardial infarction or chronic coronary heart disease. Since then, attention has been focused on its association with atherosclerotic diseases. ${ }^{10}$ A strong piece of evidence indicating a close interaction between $C$ pneumoniae infection and vascular systemic diseases was the direct detection of $C$ pneumoniae ${ }^{11}$ or the detection of heat shock proteins of $C$

pneumoniae in the plaques of the coronary and carotid arteries. ${ }^{13}$ The organisms have been shown to proliferate in vitro in vascular endothelial cells, vascular smooth muscles, and in macrophages. ${ }^{8}$ Interestingly, it has been demonstrated that macrophages infected with $C$ pneumoniae adhere to endothelial cells and transfer the pathogen to endothelial cells. Endothelial cells infected with $C$ pneumoniae show an increase expression of intercellular adhesion molecule 1 (ICAM-1) and vascular cell adhesion molecule 1 (VCAM-1), accelerating the trapping of macrophages. ${ }^{14}$ In addition, aortic sclerosis has been experimentally produced in rabbits in vivo following infection of $C$ pneumoniae through the respiratory organs. ${ }^{15}$ Thus, $C$ pneumoniae can spread to various organs. A working hypothesis is that $C$ pneumoniae interacts with the lipid metabolism through IgG antibody mediated immunoresponse in the vascular tissue which may then lead to atherosclerosis. Additionally, the potential contribution of infectious agents induced by $C$ pneumoniae has recently been clarified, and chlamydial lipopolysaccharide (LPS) or infected macrophages may produce inflammatory cytokines such as interleukin 6 (IL-6), tumour necrosis factor $\alpha(\mathrm{TNF}-\alpha)$, and matrix metalloproteinase (MMP), which impair the endothelial cells, trigger thrombus formation, and promote vascular obstruction. ${ }^{1316}$

\section{Future studies will be necessary to obtain direct evidence of $C$ pneumoniae infection by demonstrating its presence in choroidal neovascular tissue harvested during vitreous surgery}

These observations are relevant to AMD because it has been demonstrated that there is an association of the incidence of coronary heart diseases in patients with AMD. ${ }^{517}$ Recent immunohistochemical investigations have demonstrated that oxidised lipids and proteins are related to the pathogenesis of AMD. ${ }^{18} 19$ Besides, an apolipoprotein E (ApoE) gene polymorphism has been shown in $\mathrm{AMD},{ }^{20}$ and an infection by $C$ pneumoniae leads to an acceleration in the progression of atherosclerosis in ApoE deficient mice, a hyperlipidaemic animal model. ${ }^{21}$ In addition, our colleagues have reported that the serum level of oxidised low density lipoproteins (LDL) in patients with AMD was significantly higher than that in healthy controls, and that genetic polymorphism of paraoxonase, a gene involved in lipid metabolism to prevent LDL oxidation, is implicated in the pathogenesis of AMD. ${ }^{22}$

These findings strongly support the suggestion that atherosclerosis is a risk factor for AMD. Furthermore, the recent consideration of AMD as an inflammatory event was supported by the identification of several inflammation linked proteins in drusens. ${ }^{23}$

We have thus hypothesised that an infection with $C$ pneumoniae may be an additional risk factor for AMD. To test this hypothesis, we analysed the specific antibody titres of $C$ pneumoniae in the sera of patients with AMD. Informed consent was obtained from all patients after an explanation of the purpose of this study. To ensure uniformity in age distribution, the age of the patients was limited to 60-79 years. There were 27 patients with AMD (aged 71.1 (SD 6.4) years, 19 men and eight women) and 22 age matched controls (aged 69.5 (6.5) years, 12 men and 10 women). All AMD patients had the wet form of AMD which is more common in Japanese patients.

The level of IgA and IgG antibodies to $C$ pneumoniae in the serum was determined by a specific enzyme linked immunosorbent assay (ELISA) kit (Hitazyme $C$ pneumoniae, Hitachi Chemical, Tokyo, Japan) as previously reported. ${ }^{24}$ Briefly, serum samples were reacted with an outer membrane antigen of chlamydia that was purified from the YK4l strain of $C$ pneumoniae to form immune complexes with anti-human IgA or IgG antibodies. Then, p-nitrophenyl phosphate was added to the wells, and the absorbance was measured at $405 \mathrm{~nm}$. The level of IgA and IgG to $C$ pneumoniae in each sample was expressed as the IgA index and the IgG index. The mean (SD) index for 592 healthy adults has been reported to be 1.27 (0.87) for $\operatorname{IgG}$ and $1.20(0.78)$ for IgA. ${ }^{24}$

The mean index (SD) of IgG antibody for anti-C pneumoniae was 2.08 (0.95) in the AMD group and 1.32 (0.85) in the control group, while that of the IgA antibody was $1.96(0.80)$ in the AMD group and 1.39 (0.84) in the control group. Both antibody titres were significantly elevated in the AMD patients $(\mathrm{p}=0.006$ for IgG; $p=0.005$ for IgA, MannWhitney test). No significant difference 
was found between the men and women for both IgG and IgA.

We used the ELISA method to detect antibodies to the chlamydial outer membrane complex produced in infected monocytes/macrophages. Although the significance of the increased titres of specific IgG and IgA antibodies against $C$ pneumoniae is not fully understood, higher IgA and IgG antibody titres may indicate an exposure to greater amounts of $C$ pneumoniae and recurrent or chronic infections.

Increased specific titres against $C$ pneumoniae in our AMD patients suggest a possible association between AMD and C pneumoniae infection. As mentioned, although $C$ pneumoniae primarily infects the respiratory organs, $C$ pneumoniae organisms are found in the atherosclerotic lesions. Thus, macrophages infected with $C$ pneumoniae may enter the circulatory system and spread to various organs. The choroid, especially the region close to the macular area, is often the target of metastatic tumours and infections such as toxoplasmosis and histoplasmosis because the largest vascular supply is around the macular area. Therefore, we suggest that macrophages infected with $C$ pneumoniae are trapped in the vascular net in the posterior choroid and inflammatory cytokines such as TNF- $\alpha$ and MMP can be produced. These agents impair vascular architecture and even trigger a rupture of choroidal vessels, which may help in the development of AMD.

Other than AMD, infection with $C$ pneumoniae has been proposed as a risk factor for ischaemic optic neuropathy. Two research groups have investigated this association, but different results were reported; one showed significantly higher IgG titres in patients with ischaemic optic neuropathy, ${ }^{25}$ and the other reported no significant differences from the controls. ${ }^{26}$

The limitations of our study are that it was a retrospective study of a small sample and combined systemic factors were not matched. However, because AMD might be associated with various systemic vascular disorders, a higher incidence of infection with $C$ pneumoniae seen in AMD patients should be noted. Future studies will be necessary to obtain direct evidence of $C$ pneumoniae infection by demonstrating the presence of $C$ pneumoniae in choroidal neovascular tissue harvested during vitreous surgery. Our findings do not necessarily justify $C$ pneumoniae infection as the primary cause of AMD. However, inflammatory events caused by a focal infection around the macular area with $C$ pneumoniae might affect the surrounding atherosclerotic environment as an additional factor. The association between a pathogen and AMD is worth further investigation because a new approach using antibiotics might treat this incurable vision threatening disease.

Br J Ophthalmol 2003;87:523-524

\section{Authors' affiliations}

O Ishida, H Oku, T Ikeda, Osaka Medical College, Osaka, Japan

M Nishimura, Kyoto Prefectural University of Medicine, Kyoto, Japan

K Kawagoe, Hitachi Chemical Co Ltd, Japan K Nakamura, Nakamura Eye Clinic, Japan

Correspondence to: Hidehiro Oku, Osaka

Medical College, Osaka, Japan;

hidehirooku@aol.com

Accepted for publication 21 December 2002

\section{REFERENCES}

1 Cruickshanks KJ, Klein R, Klein BEK. Sunlight and age-related macular degeneration: The Beaver Dam Eye Study. Arch Ophthalmol 1993;111:514-18.

2 Vingerling JR, Hofman A, Grobbee DE, et al. Age-related macular degeneration and smoking. The Rotterdam Study. Arch Ophthalmol 1996;114:1193-6.

3 Snodderly DM. Evidence for protection against age-related macular degeneration by carotenoids and antioxidant vitamins. Am J Clin Nutr 1995;62(6 suppl):S1448-61.

4 The Eye Disease Case-Control Study Group. Risk factors for neovascular age-related macular degeneration. Arch Ophthalmol 1992;110:1701-8

5 Vingerling JR, Dielemans I, Bots ML, et al. Age-related macular degeneration is associated with atherosclerosis. The Rotterdam study. Am J Epidemiol 1995; 142:404-9.

6 Mares-Perlman JA, Brady WE, Klein R, et al. Dietary fat and age-related maculopathy. Arch Ophthalmol 1995;113:743-8.

7 Grayston JT, Kuo CC, Wang SP, et al. A new Chlamydia psittaci strain, TWAR, isolated in acute respiratory tract infections. $N$ Engl J Med 1986;315:161-8.

8 Gaydos CA, Summersgill JT, Sahney NN, et al. Replication of Chlamydia pneumoniae in vitro in human macrophages, endothelial cells, and aortic artery smooth muscle cells. Infect Immun 1996;64:1614-20.

9 Saikku P, Leinonen M, Mattila K, et al. Serological evidence of an association of a novel Chlamydia, TWAR, with chronic coronary heart disease and acute myocardial infarction. Lancet 1988;2:983-6

10 Kalayoglu MV, Libby P, Byme GI. Chlamydia peumoniae as an emerging risk factor in cardio vascular disease. JAMA 2002;288:2724-31.

11 Kuo CC, Shor A, Campbell LA, et al. Demonstration of Chlamydia pneumoniae in atherosclerotic lesions of coronary arteries. J Infect Dis 1993;167:841-9.

12 Campbell LA, O'Brien ER, Cappuccio AL, et al. Detection of Chlamydia pneumoniae (TWAR) in human coronary atherectomy tissues. J Infect Dis 1995; 172:585-8.

13 Kol A, Sukhova GK, Lichtman AH, et al. Chlamydial heat shock protein 60 localizes in human atheroma and regulates macrophage tumor necrosis factor-alpha and matrix metalloproteinase expression. Circulation 1998;98:300-7.

14 Kalayoglu MV, Perkins BN, Byme GI. Chlamydia peumoniae-infected monocytes exhibit increased adherence to human aortic endothelial cells. Microbes Infect 2001;3:963-9.

15 Fong IW, Chiu B, Viira E, et al. Rabbit model for Chlamydia pneumoniae infection. J Clin Microbiol 1997;35:48-52.

16 Quinn TC, Gaydos CA. In vitro infection and pathogenesis of Chlamydia pneumoniae in endovascular cells. Am Heart J

1999;138:S507-1 1.

17 Chaine G, Hullo A, Sahel J, et al. Case-control study of the risk factors for age related macular degeneration. France-DMLA Study Group. Br J Ophthalmol 1998;82:996-1002

18 Ishibashi T, Murata T, Hangai M, et al. Advanced glycation end products in age-related macular degeneration. Arch age-related macular degeneration.
Ophthalmol 1998;1 16:1629-32.

19 Handa JT, Verziil N, Matsunaga H, et al. Increase in the advanced glycation end product pentosidine in Bruch membrane with age. Invest Ophthalmol Vis Sci 1999;40:775-9.

20 Schmidt S, Saunders AM, De La Paz MA, et al. Association of the apolipoprotein $\mathrm{E}$ gene with age-related macular degeneration: possible effect modification by family history, age, and gender. Mol Vis 2000;6:287-93.

21 Moazed TC, Campbell LA, Rosenfeld ME, et al. Chlamydia pneumoniae infection accelerates the progression of atherosclerosis in apolipoprotein E-deficient mice. J Infect Dis 1999; 180:238-41

22 Ikeda T, Obayashi $H$, Hasegawa G, et al. Paraoxonase gene polymorphisms and plasma oxidized low-density lipoprotein level as possible risk factors for exudative age-related macular degeneration. Am J Ophthalmol 2001;132:191-5.

23 Anderson DH, Mullins RF, Hageman GS, et al. A role for local inflammation in the formation of drusen in aging eye. Am Ophthalmol 2002;134:411-31.

24 Kishimoto T, Matsushima T, Morikawa T, et al. Assay of specific anti-chlamydia pneumoniae antibodies by ELISA method. 3 . Setting of serological criteria. J Jpn Assc Infect Dis 1999;73:457-466. (in Japanese)

25 Wegar M, Haas A, Stanger O, et al. Chlamydia pneumoniae seropositivity and the risk of nonarteritic ischemic optic neuropathy. Ophthalmology 2002; 109:749-52.

26 Vécsei PV, Kircher K, Reitner A, et al. Chlamydia in anterior ischemic optic neuropathy. Ophthalmologica 2002;216:215-20. 